\title{
Monitoring pavement relief of the Hume Highway during undermining
}

\author{
GE Swarbrick Pells Sullivan Meynink, Australia \\ P Meers Roads and Maritime Services, Australia \\ D Lee Shoy Roads and Maritime Services, Australia \\ DJ Kay Mine Subsidence Engineering Consultants, Australia
}

HG Buys AECOM, Australia

\begin{abstract}
The Hume Highway (M31) is arguably Australia's most important piece of transport infrastructure, carrying around 45,000 cars, trucks and bases per day, which equates to AUD 22 B worth of goods each year.

In 2006, it was proposed to undermine the Hume with a series of long wall panels near Douglas Park, NSW, which would obliquely undermine the dual carriageway causing subsidence of up to $1 \mathrm{~m}$. At the time the potential economic loss due to mining impacts on M31 without mitigation was estimated to be around AUD 125 M. Six years later, the fourth longwall panel is nearing completion while the mitigation strategies have allowed the highway to operate continuously without incident and with minimal delays to traffic.

Key to the success of the project is the ability to accurately measure the response of the pavement under mining induced strains and the mitigatory pavement compression relief mechanisms. A system of around 600 optical fibre Bragg gratings (FBG) are used to monitor changes in pavement strain at $10 \mathrm{~m}$ intervals above the workings. The increases in compressive strain are subsequently relieved by closure within a network of 80 slots, each pre-constructed transversely through the full depth of the pavement structure and fitted with crackmeters to measure closure. Additional monitoring elements include crack meters to measure differential expansion across bridge joints, a shape array to monitor any shear movement below the base of the Douglas Park Bridges and manual inclinometers and survey marks.
\end{abstract}

The success of the monitoring system has provided the ability to correlate changes in ground strain, pavement strain and strain relief due to slot closure. This paper provides an overview of the system with a focus on the way in which strains and movements are evaluated and verified across alternate measuring systems.

\section{Introduction}

The Hume Highway (M31) links Sydney with Canberra and Melbourne and is the busiest freight road corridor in the country. The dual two-lane carriageway currently carries in excess of $34 \mathrm{M} \mathrm{t}$ of road freight annually with an estimated goods value of AUD $22 \mathrm{~B}$. The M31 in relation to longwalls is shown in Figure 1.

Mining was first proposed in 2005 with six longwalls to be situated directly below M31 each being $320 \mathrm{~m}$ wide (rib-to-rib), about 3 to $3.3 \mathrm{~m}$ in thickness and at around 500 to $600 \mathrm{~m}$ below the surface. The location of longwalls LW702 to LW705 is shown in Figure 1. Longwall LW706 is currently in operation and located immediately to the north of LW705.

Consultation between the mine operator and the Roads and Traffic Authority of NSW lead to the formation of a technical committee charged with the challenge of developing measures to manage potential impacts of subsidence on highway infrastructure, including pavements, culverts, cuttings and bridges. The technical committee included the mine operator, Roads and Maritime Services, and other stakeholders and 
specialists in the fields of pavement and bridge engineering, geotechnical engineering, subsidence, surveying and monitoring systems, project management and traffic management.

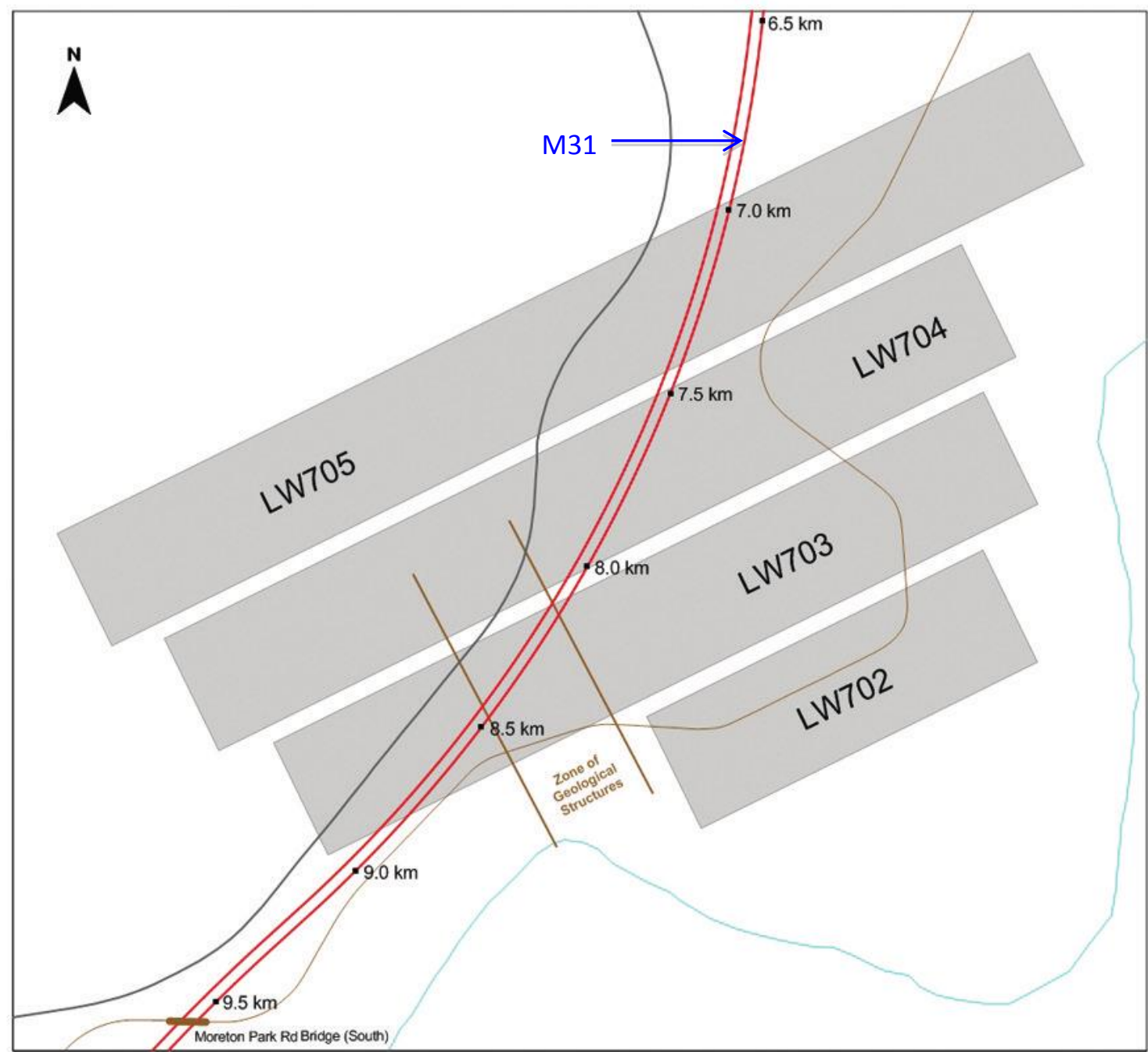

Figure 1 Location of M31 and longwalls LW702 to LW705

\section{Subsidence impacts}

The initial studies into potential mining impacts on the Hume Highway were commenced in 2005. This initial work was undertaken by mine subsidence engineering consultants (MSEC) and comprised empirical predictions of subsidence, tilt, strain and curvature across the site.

MSEC used incremental profile methods developed in-house to predict impacts including extensive adjustments for closure, upsidence (reduced subsidence in valleys), adjacent panels, sequencing, proximity to valleys and other influences.

Given the proximity of proposed mining to M31 and other key infrastructure (i.e. the Douglas Park twin bridges) the key prediction challenges faced by MSEC were:

1. Travelling or transient impacts associated with the approach and subsequent retreat of longwalls beneath the roadway.

2. The magnitude of far field (regional movements) on nearby infrastructure and the Douglas Park twin bridges in particular.

3. The extent of stress and strain that would be transferred from the softer natural ground into the stiffer overlying pavement. 
At the time of the initial MSEC predictions, much was understood about the mechanism and approximate magnitude of far field (also known as regional) measurements. However, little was known of the statistical likelihood of magnitude of far field movements with respect to distance. This prompted a major study of far field movements in the Southern Coal Fields and the assessment of the risk of potential impacts on the Douglas Park twin bridges and other infrastructure was significantly improved.

The most challenging aspect of the impact assessment was the expected behaviour of the pavement in response to subsidence induced compression. Experience with pavements undermined elsewhere and the F6 freeway in particular suggested that compression (shear) failure leading to a step in the pavement of 40 to $80 \mathrm{~mm}$ high was possible in response to undermining. Subsequent risk assessments by the road authority suggested that a step in the pavement of only $50 \mathrm{~mm}$ could be sufficient to lead to severe injury and even death of a road user, particularly a motorcycle rider. Therefore, the measurement and mitigation of compression related pavement impacts became a focal point for the approval of M31 undermining.

More details on the background and studies undertaken for the assessment of the M31 pavement and likely impacts are provided in Kay et al. (2011) and Buys et al. (2014).

\section{$3 \quad$ Impact mitigation by pavement slotting}

The measured pavement characteristics in combination with MSEC subsidence predictions indicated that without modification the M31 would potentially experience high compressive strains in some areas. These strains were deemed to be potentially high enough to cause localised failures and steps at the pavement surface that could render the road unsafe.

The technical committee (TC) investigated a number of alternatives for preventing compressing stepping and eventually selected the use of slots installed through the full depth of the pavement structure as the primary mitigating measure. The choice of slots was largely based on experience with the use of asphaltic concrete (AC) plugs to span bridge joints (e.g. Park et al. 2011; 2010). It was estimated that a slot $100 \mathrm{~mm}$ wide extending across the entire pavement width and through the stiffer underlying subgrade would allow the predicted compressive strains to be relieved and kept below safe limits. A structural bridge (a fibre cement sheet) over the slot would allow the upper AC to be reinstated and traffic to use the road as normal. A schematic of the slot design is shown in Figure 2.

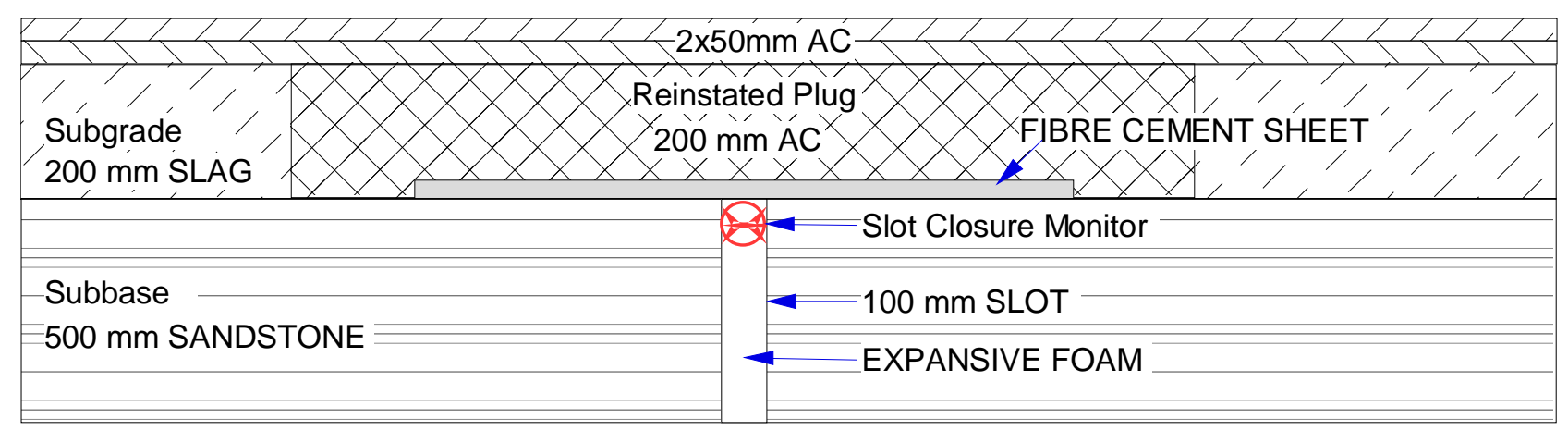

\section{Figure 2 Pavement slot design}

Initially sixteen slots were cut in the pavement (eight in each carriageway) directly above Longwall LW703 installed during partial road closures. The spacing between slots were based on subsidence predictions by MSEC and global numerical modelling with extra slots added within a zone of geological structures identified by Pells Sullivan Meynink (PSM) as part of their geotechnical study.

During the initial works the method of construction was refined and improved such that the entire process of road closure, slot installation (including monitoring devices) and road reinstatement could be undertaken in one shift. This was based on the need to install slots within restricted road closure periods and, importantly, overnight to minimise traffic disruption. More details on slot design and installation may be found in Kay et al. (2011). 


\section{$4 \quad$ Monitoring systems}

The success of the pavement slotting system was contingent on a robust, accurate, comprehensive and prompt means by which the pavement strain could be monitored. Key requirements of such a system were:

- Accuracy - measurements would need to reliably detect small strains within a live pavement of around $0.1 \mathrm{~mm} / \mathrm{m}(100 \mu \mathrm{s})$.

- Response time - readings would need to be detected, analysed and distributed within, potentially, a few hours and preferably in real time.

- Stability - the system would need to provide reliable readings over the expected period while under any climate or traffic conditions.

- Longevity - the devices would need to be installed well ahead of mining due to the planning and logistics involved in closing such a major motorway.

- Cost-effectiveness - at least $600 \mathrm{~m}$ of pavement would need to be monitored continuously and potentially increased to around $2.5 \mathrm{~km}$ to accommodate additional longwalls.

At the time the only method that appeared to potentially satisfy these criteria were optical fibre systems. Two were subsequently considered for further investigation:

- Optical time domain reflectometry (OTDR).

- Fibre Bragg gratings (FBG).

OTDR is a well-established technique where an optical signal is sent along an optic fibre and the reflection or reflections measured. The timing and phase change of reflected signals can potentially detect:

- Breaks in the cable.

- Kinks or strains.

OTDR measures over its entire length making it an attractive means of monitoring the entire pavement.

FBGs have a periodic variation in the refractive index of the fibre core at specified locations. These variations reflect light with a phase shift determined by the period of variation. Originally, FBGs were designed to filter out specific wavelengths. It was subsequently discovered that the change in reflected wavelength could be calibrated to strain and/or temperature. Typical FBG sensitivities to strain and temperature are $1 \mathrm{~nm} / 1,000 \mu \varepsilon$ and $1 \mathrm{~nm} / 100^{\circ} \mathrm{C}$. A phase shift example is shown in Figure 3 .

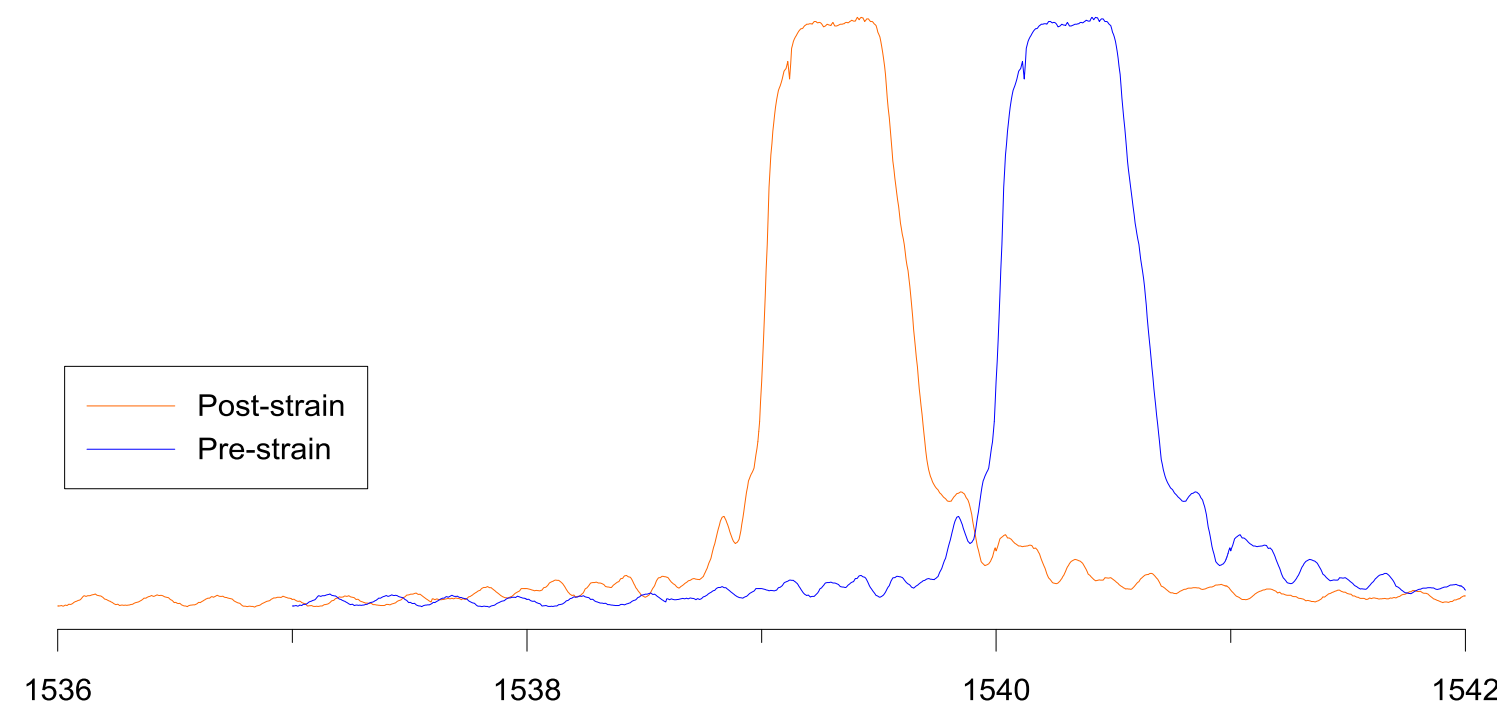

Figure 3 Example phase shift reflected by an FBG 
Each single FBG is etched into the core of the optic fibre using a high-energy UV laser. FBGs can be concatenated onto a single optic fibre with each having their own signature wavelength response. In this way a string of FBGs can be created which can be read using one interrogator which sends a range of frequencies as shown diagrammatically in Figure 4. The number of FBGs that can be concatenated depends on the expected range of strain and hence the expected shift in wavelength.

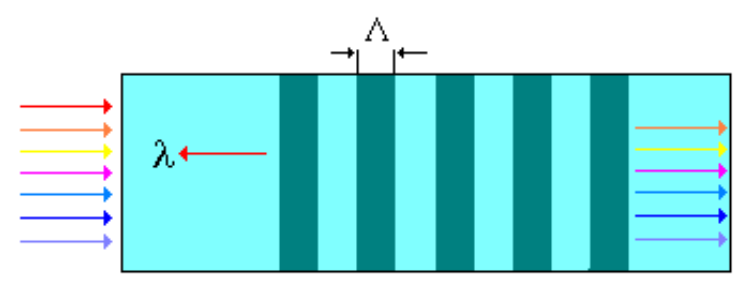

\section{Figure 4 Selective reflection of a wavelength range by a specific FBG}

Recognised drawbacks for optical systems are:

- Optical fibre cables are difficult to repair once they are installed, particularly on-site. When breaks occur optic fibres can be interrogated from the alternate end. However, multiple breaks require additional monitoring locations to be established.

- FBGs have a limit to the number of sensors that can be installed on any one segment. The maximum number of sensors on a cable is limited by the bandwidth capacity of the interrogator and the expected range of strains, so that the central wavelengths for each sensor do not overlap during the monitoring period. OTDR does not have these bandwidth limitations.

- Permanent power and communications are required for all automated monitoring systems. The interrogation requirements for optic fibre systems are also relatively expensive which also affects security costs.

At the time, there were several known applications where OTDR had been used to detect strain and movement within pavements. However, there were potential issues with OTDR longevity and therefore it was decided to use both OTDR and FBG systems in tandem as a complementary redundancy measure. At the time, there were no known applications of FBG in pavements anywhere in the world. There was, however, some experience with the use of FBGs to measure strains on related structures such as bridges.

A number of studies were commissioned using OTDR and FBGs in combination to ascertain their use for the M31 project. These studies included:

- Laboratory studies to assess the most efficient means of installation such as depth within the pavement and method of bonding to ensure longevity under high and frequent traffic loading.

- Field trials along a $90 \mathrm{~m}$ section of a rural roadway subject to live traffic loading and subsidence from longwall mining direct below the test site.

- Field trial of two $0.9 \mathrm{~mm}$ OTDR cables installed in a section of M31 pavement during mining in an adjacent longwall.

In particular, the field trials demonstrated that:

- The installation method was appropriate (epoxy grouted in a $20 \mathrm{~mm}$ deep trench $8 \mathrm{~mm}$ wide).

- The FBG cables could reliably and accurately (when compared to measured ground strain) record strain under live traffic for pavements subject to $440 \mathrm{~mm}$ of subsidence.

- The OTDR could provide a continuous measure of pavement bending, compression, stretching and breakage; however, the $0.9 \mathrm{~mm}$ cables showed evidence of fatigue failure and thicker cables should be considered. 
- Temperature induced strains were a significant contributor to total strain and need to be accounted for.

Importantly the field trail demonstrated the need to correct FBG strains for temperature. For the M31, the expected range of strains for the pavement was $\pm 2 \mathrm{~mm} / \mathrm{m}(2,000 \mu \varepsilon)$. Each FBG would be significantly affected by temperature effects of $10 \mu \varepsilon$ change in strain per ${ }^{\circ} \mathrm{C}$. Consequently FBGs installed in M31 were used in combination - one coupled and uncoupled. The Uncoupled FBG reacts only to strain while the coupled FBG reacts to both strain and temperature. By combining both readings for the same location, both temperature and strain can be measured. However, this limits the total number of FBGs that could be interrogated on a single optic fibre to 10 based on the spectrum of light used for interrogation.

More details on these trials other studies on FBG in particular can be found in Kay et al. (2007) and Nosenzo et al. (2013).

The limited experience of these optical methods in pavements meant that they had to be supplemented by established techniques such as traditional survey. Traditional surveys were also initially adopted as the primary triggers. In summary, the main monitoring systems to protect the M31 included:

- Ground survey - absolute 3D and 2D surveys undertaken at weekly or twice weekly intervals during active subsidence. Separate monitoring lines were located in the ground adjacent and alongside both carriageways.

- OTDR cables - four OTDR cables were installed along lengths of potentially affected carriageway. These comprised two $0.9 \mathrm{~mm}$, one $1.6 \mathrm{~mm}$ and one $2.9 \mathrm{~mm}$ cables. The thicker 1.6 and $2.9 \mathrm{~mm}$ cables were used for longwalls after LW703 due to the fatigue breakage of the $0.9 \mathrm{~mm}$ cables found during trials. Evidence of breakage or strain were monitored and uploaded continuously.

- FBG sensors - FBG sensors were installed every $10 \mathrm{~m}$ in both carriageways. Pavement strains were measured and uploaded every 15 minutes.

- Slot displacement sensors - opening and closure of each slot was measured by vibrating wire displacement sensors (crackmeters) with readings taken and uploaded every five minutes.

- Visual inspections - Comms Network Solutions (CNS) undertook detailed visual inspections on a weekly or twice weekly basis particularly during mining under/adjacent to M31. Vehicle-based patrols were undertaken by NSW Police each day and by RMS each day during peak periods.

- Inclinometers - inclinometers were placed in a fault that was identified in a railway cutting (fixed in-place inclinometer) and below the Douglas Park twin bridges (two inclinometer casings read manually). Readings were taken and uploaded every 15 minutes for the in-place inclinometer and weekly or monthly for the manual inclinometers depending on proximity to mining.

- Bridge deck joint monitors - the movement between Douglas Park bridge deck segments was monitored across twelve joints also using vibrating wire displacement sensors (crackmeters). Readings were recorded and uploaded every 15 minutes.

- Visual inspections - visual inspections were undertaken by at least two independent entities. There were typically undertaken and reported daily and as directed by the TC.

Systems were installed over a length of pavement typically around three times the length directly undermined.

The monitoring system was coordinated and managed through a central database that could be continually accessed by the TC and others. The database included both raw and processed data and the ability to produce instantaneous graphs. Several components of the system were alarmed with automated text messaging, emails and dedicated communication links to $24 \mathrm{hr}$ emergency control stations. 


\section{$5 \quad$ Monitoring performance}

\subsection{Data analysis, assessment and reporting}

Monitoring data was collated, analysed and summarised, then reviewed by the TC. Data analysis was undertaken by designated consultants using procedures and techniques directed or approved by the TC. In many cases new calibration, analysis and presentation methods had to be developed to satisfy the requirements of the TC and other stakeholders.

Reports were created weekly during mining with key personnel monitoring data and trends on a daily and sometimes continual basis. The weekly reports provided an analysis of results, identified trends or irregularities in the data, compared the results with predictions (where relevant) including back analysis for continuous improvement, assessed or forecast exceedance of triggers and recommended further actions as required. Any anomalous movements or unusual trends were communicated to the TC as soon as they were detected for further consideration, tracking, and evasive action.

Further actions directed by the TC included immediate inspections by designated personnel, undertake additional slotting of the pavement (including additional sensors), repair pavement damage (including milling of steps or raised areas) and repair of monitoring equipment damage where possible. Traffic management included variable message signs that could be activated and configured remotely to enact localised speed restrictions or provide warnings.

\section{$5.2 \quad$ OTDR performance}

The OTDR cables were installed in early 2008 about ten months before the commencement of LW703. During direct undermining of M31 by longwall LW703 the $0.9 \mathrm{~mm}$ OTDR cables exhibited increasing signal loss and breakages due to fatigue requiring repair. The 1.6 and $2.4 \mathrm{~mm}$ cables remained in operation; however, these also suffered increasing signal loss over time.

Post LW703 a review of the monitoring system found that the OTDR detected pavement deformations rapidly and before being detected by FBGs and that this was achieved at a relatively low incremental cost. However, OTDR signal loss increased over time increased noise and generated false alarms. Several reductions in sensitivity were required to avoid these alarms leading to an overall reduction in the reliable resolution for detecting pavement deflection.

Overall, the OTDR cables were found to be useful in terms of corroborating movements and strains detected by other systems. However, they did not exhibit sufficient robustness, accuracy or stability to warrant their use as the primary monitoring system.

\subsection{FBG performance}

FGBs have now been in operation within the M31 pavement since March 2009, initially for the purposes of calibration and establishing a baseline data, then for monitoring pavement impacts when undermining commenced in August 2010. Currently longwall LW706 has progressed well past the motorway and the vast majority of FBGs continue to provide accurate strain and temperature data. During the six year intervening period the FBGs have demonstrated the following:

- Measurements of compressive strain to an estimated overall accuracy of around $0.01 \mathrm{~mm} / \mathrm{m}$ $(10 \mu \varepsilon)$.

- Survivability over a considerable time period continued and often under heavy traffic loading, large temperature fluctuations of up to $40^{\circ} \mathrm{C}$, extended periods of wet and dry climate and lightning strikes.

- An ability to be repaired, extended or accessed from alternate locations within the optic fibre.

- Rapid response being normally interrogated every 15 minutes but capable of measurement in essentially real-time. 
The longevity and robustness of the system has enabled a number of related studies to be undertaken, namely the change in pavement strain with temperature, the relationship between pavement strain and adjacent ground strain and the performance of pavement slots before and after installation. More details of these studies may be found in Buys et al. (2014).

These advances were not, however, achieved without some important lessons learnt along the way. Two of these were the need for in-place calibration and behaviour under tensile strain as discussed below. Also discussed below is the observed relationship between pavement and ground strain.

\subsubsection{In-place calibration}

During the ten months or so prior to undermining, the FBGs were monitored continuously (i.e. every 15 minutes) to assess longevity and stability. During this period, the strain and temperature readings were compared to confirm the theoretical linearity of instrument measurement and to obtain individual temperature correction factors. While this expectation proved to be correct for the majority of FBGs there were several that did not conform to theory. Instead of a linear relationship these nonconforming FBGs produced a bilinear response whereby there was an increase in strain (i.e. tension) with increasing temperature at low temperatures followed by a decrease in strain with increasing temperature at higher temperatures. These so-called 'boomerang' calibration relationships were observed for about $30 \%$ of all FBGs.

The reason for this non-linearity is not well understood. It has been postulated that this behaviour is possibly related to differences in pavement type or other local factors. The most likely explanation is the presence of cracks within the pavement that create a non-linear response whereby increasing tension is not detected by the FBGs due to lack of strain transference. Further discussion of these effects is provided in Section 5.3.2.

Regardless of the cause, the effect of a bilinear response is that calibration curves need to be created in situ for each FBG. This is reliant on sufficient installation time such that the majority of temperature change can be experienced prior to mechanical (not temperature related) impacts on strain. In additional this finding means FBGs should always be installed as a strain-temperature pair to facilitate this calibration. In the case of M31 the initial period of around 10 months was considered to be a minimum. Subsequent extensions of the FBG network to cover additional longwalls were undertaken well in advance of subsidence impacts.

\subsubsection{Tensile strain}

FBGs were not found to be reliable for detecting pavement tensile strain. This is due to the way the pavement responds to tensile strain whereby movement is concentrated on existing cracking that has occurred under normal pavement function. The FBGs measure over the length of the sensor, typically 5 to $10 \mathrm{~mm}$, and are therefore unlikely to be located at the locations of concentrated strain and therefore do not reliably detect these movements. At the same time if the FBG was to be located on a crack or other relief point the concentrated tensile strain may be more likely to exceed the instrument limits and potentially break the optic fibre.

The experience at M31 was as described above with FBGs deemed unreliable when the pavement was in tension. Installation of FBGs has been improved such that crack mapping is used to ensure that FBGs are not installed at known locations where tensile strain would concentrate. However, in this particular application, all of the triggers and controls are based on knowledge of compressive strain only.

\subsubsection{Active measurement of pavement response}

For the majority of their use the FBGs were used in a 'passive' monitoring capacity whereby the pavement response was recorded as a reaction to temperature and ground strains. There were also at least two means by which the pavement was 'actively' monitored - that is, used to measure pavement response under actively applied stresses or pavement changes. 
The first of these applications was the use of FBGs to record pavement response under the direct application of load to the pavement surface. Loads were applied through the use of a heavy vehicle whose tyres were positioned directly over each FBG. The response of each FBG was subsequently recorded and position confirmed. The purpose of this exercise was to confirm the correct installation of FBGs in terms of both position and expected wavelength response. This procedure was not employed initially but introduced in subsequent longwalls in response to an error in the FBG identification and location. Since introduction, this procedure has become a standard component of FBG quality assurance for this project.

The second 'active' measurement application involved monitoring the real-time response of the pavement during the installation of additional slots. Additional slots were installed during active undermining in response to build-up of compression strain. Slot need, timing and location was at the direction of the TC.

During installation, the change in pavement stress due to slotting was measured in real-time by nearby FBGs. The FBGs were sensitive enough to detect vehicle movements, milling of the surface AC, strain relief due to cutting the vertical slot and even thermal expansion due to surface heating during resealing.

In most cases, the closure in response to slotting (measured by surface survey) correlated very closely to the measured reduction in compressive strain. Furthermore, the lateral extent of strain relief by slotting was accurately captured by the FBGs. Examination of this data informed the TC on:

- The rate at which slotting relieved pavement stress.

- What length of pavement could be relieved by pavement slots.

- The overall effectiveness of stress relief by comparison of strain reduction with slot closure.

- Estimates of mechanical parameters that control pavement closure, such as base friction.

An example of the observed response to pavement slotting is shown in Figure 5 . The effect of construction and even heat expansion of the pavement during the application of AC can be observed within the FBG record. This is also shown in the observed correlation between the strain relief recorded by the FBGs and the closures recorded by ground survey pins monitored before and after slotting and the slot closure monitor.

From Figure 5, it is evident that for this particular slot:

- Immediate strain relief was observed at sensor S5.3 up to $35 \mathrm{~m}$ away (left hand side) when the new slot SB00 was cut.

- Immediate strain relief between new slots SBO0 and SB00.5 and between new slot SB00.5 and pre-cut slot SB01 was observed when these new slots were cut. The spacing between these slot pairs is around 60 and $70 \mathrm{~m}$, respectively.

- There was greater strain relief within sections of pavement at closer slot spacing with effectively the entire pavement between SB00.5 and SB01 being relieved over the entire $70 \mathrm{~m}$ slot spacing, i.e. $35 \mathrm{~m}$ away from any slot.

- The majority of strain relief occurred within the first hour of slotting.

The immediacy at which strain is relieved is an important consideration. On one occasion, machinery became caught within the slot during cutting requiring a review of procedures.

These observations were fairly typical of the measured response of slots cut during mining with some variation in the affected length of pavement and response time. 
FBG Line - Southbound

$$
\underset{\sum \infty}{\leftarrow \infty} \begin{aligned}
& \text { Slots installed } \\
& \text { during mining }
\end{aligned}
$$

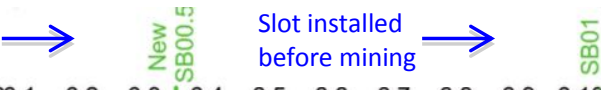

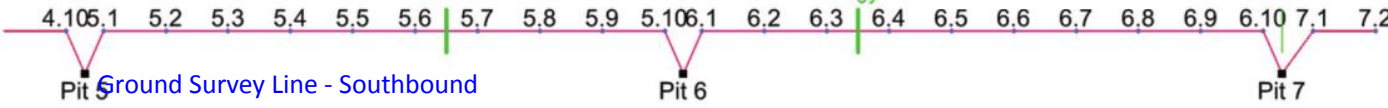

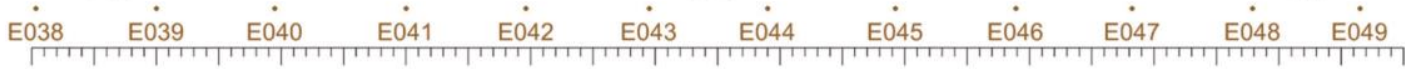

\section{惫息} Highway Chainage $(\mathrm{km})$
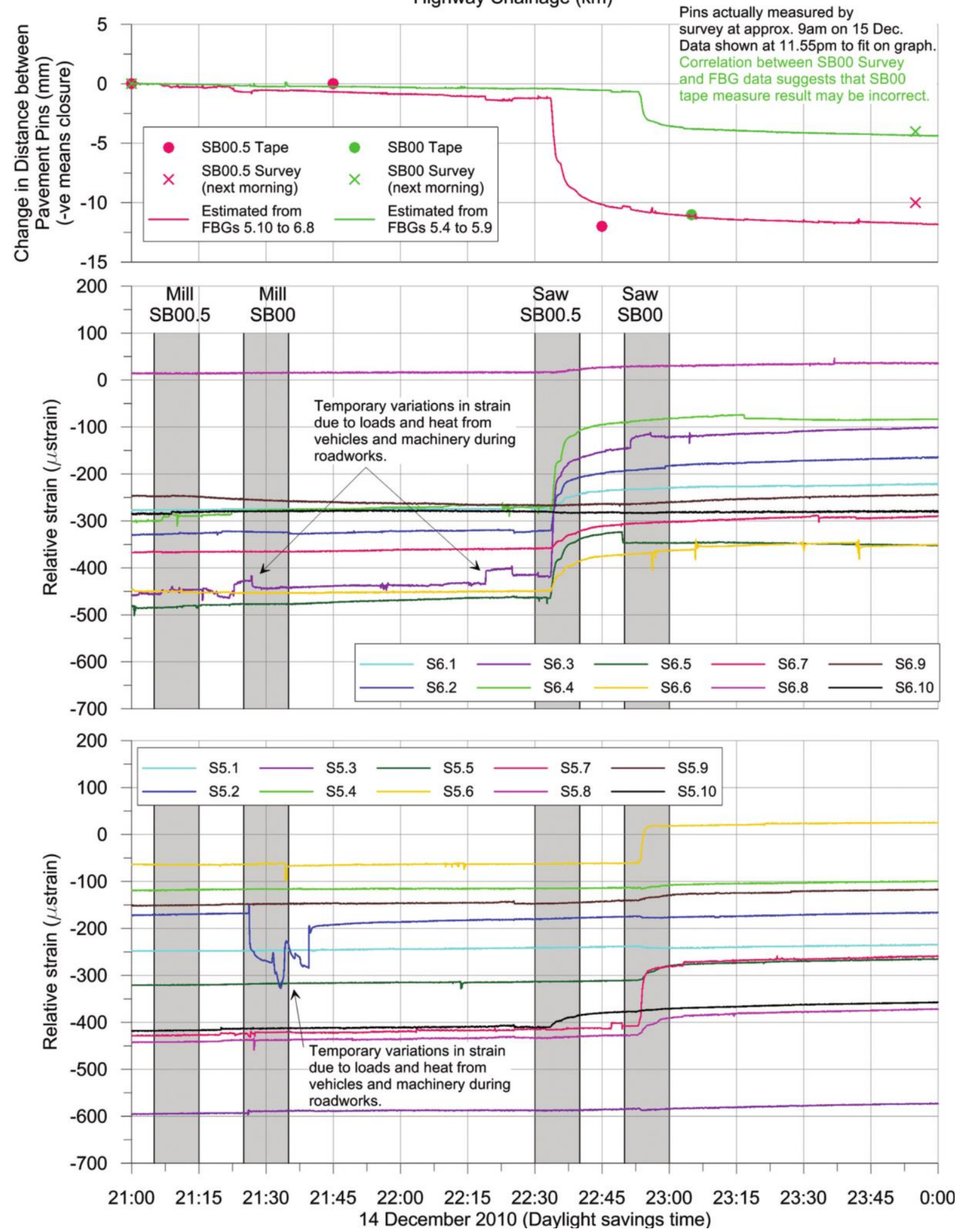

Figure 5 Real-time response of FBGs during installation of new slots SB00 and SB00.5 


\subsubsection{Coalescence}

As discussed above, the FGBs for M31 were designed to accommodate up to $2 \mathrm{~mm} / \mathrm{m}$ allowing ten to be used per interrogated segment. If this limit is exceeded the phase-shift is such that the returned signal coalesces with an adjacent FBG signal (either higher or lower wavelength depending on whether the exceedance is in tension or compression). This occurred in at least one location preventing automated measurement for the two affected FBGs, i.e. the FBG for which the nominal strain limit had been exceeded and the FBG into which this signal had coalesced.

However, the FBG supplier, Monitor Optics, was able to separate the coalescing signals by careful examination of the timing and phase shift of the reflected signals. This technique cannot be readily automated and allowed the TC to retain key information on pavement performance, particularly where strain limits have been exceeded.

\subsubsection{Pavement versus ground strain}

Initially triggers that regulate the installation of additional pavement slots, speed restrictions and even road closures were all based on ground strains from traditional survey methods, this being in line with regulatory requirements. However, no reliable means for measuring pavement strain using traditional survey could be established. Therefore, empirical methods were used to estimate pavement strain from ground strain, measured by traditional survey.

From observations over time, it was established that the M31 pavement strain measured by the FBGs was at most about one half of the ground strain measured by traditional survey. This compares to the initial field trial in a rural road where the relation between ground and pavement strain was approximately 1 to 1 . However, the M31 pavement is stiffer than that used in the trial and therefore the pavement strain response was found to be consistently significantly less than that measured in the ground.

After several years of data and the successful management of two longwalls undermining M31, the TC determined that the FBG readings could be used as a primary trigger. The regulator subsequently approved a request by the $\mathrm{TC}$ to use FBG data as the primary trigger measure in place of ground survey. This trigger method has been in operation for the past two longwalls.

Examination of changes in pavement and ground strain has generally been found to correlate well with slot closure. An example of this correlation is shown in Figure 6.

In Figure 6, the total closure (shown as a continuous black line) between pre-cut slots NB01 and NB02 has been calculated from half the combined slot closure (assumed to be shared between adjoining pavement sections) and the sum of all recorded FBG sensors (40.1 to 40.10). This has been compared to the weekly ground survey (green triangles). Also shown is the closure recorded from a new slot cut during mining around 5 February 2011, NB07.5.

From Figure 6 it is apparent that for this record:

- There was a good correlation between computed total strain and measured ground strain for both the north and south carriageways.

- Slot cutting had an immediate impact on FBG strains in the northbound pavement which was approximately matched by the slot closure resulting in no change to total strains.

- The ground strains were not affected by slotting as expected.

- The southbound strains did not result in any significant slot closure which is likely to be due to the lower strain experienced by the pavement in this area in combination with the relatively large distance between slots of around $100 \mathrm{~m}$.

- The small decrease in strain recorded in the southbound carriageway in November 2010 is due to the effect of the travelling wave, which resulted in a reversal from tension to compression as undermining progressed below the carriageway. 


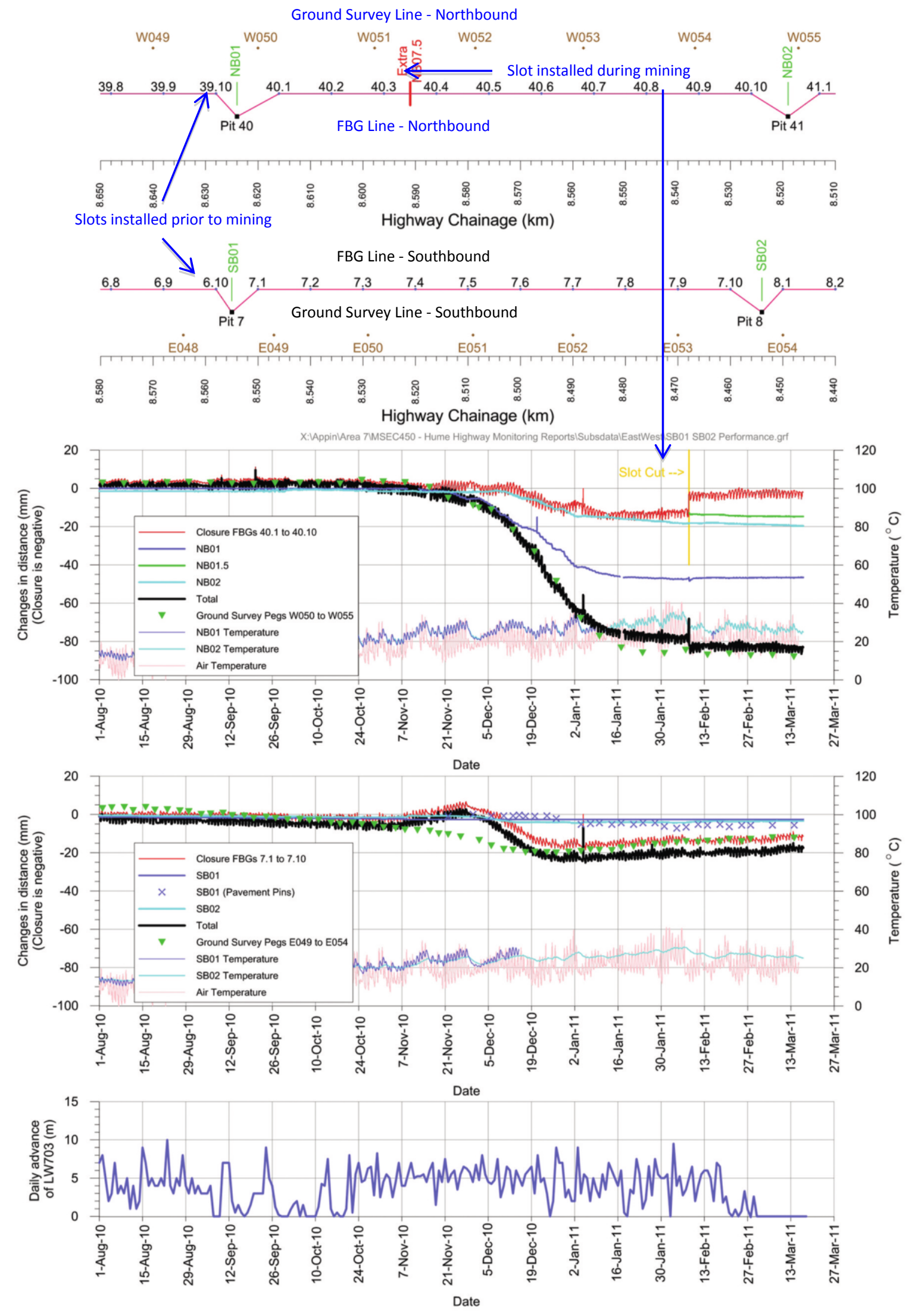

Figure 6 Correlation of ground survey, FBG strains and slot closure during undermining 
Buys et al. (2014) has undertaken a more in-depth analysis of the relationship between pavement strains including the effect of temperature. Buys et al. (2014) also provides some specific examples of anomalous movements that were recorded during undermining and the remedial measures undertaken.

\subsection{Vibrating wire displacement sensors}

Slot and bridge joint movements were monitored using vibrating wire displacement sensors (crackmeters) with a travel of at least $60 \mathrm{~mm}$ for the slots and around $10 \mathrm{~mm}$ for the bridge joints. These sensors have demonstrated good stability since installation with little sign of drift or measurement error.

Of the 81 slot sensors, all but one sensor are still accessible while two bridge joint sensors have been replaced. The sensors also record temperature, which is readily comparable with FBG data.

\section{Conclusion}

The success of the pavement slotting system was contingent on a robust, accurate, comprehensive and prompt means by which the pavement strain could be monitored. A range of techniques was employed as part of the management plan for the undermining of M31. Of these techniques, FBGs were found to be the most appropriate means of monitoring the pavement slotting system due to their accuracy, stability, response time and longevity. They were also found to be cost effective; however, no other single system was known that could fulfil the monitoring requirements irrespective of cost.

The use of FBGs was not without some technical difficulties, these mainly relating to calibration, unsuitability for measuring pavement tension, efficient repair after damage, bandwidth limitations and the interrogation requirements including power and site security. Of particular importance was the need to install FBGs as a strain-temperature pair and monitor as long as possible prior to active use. This allows seasonal and other variations to be observed and an in-place calibration established.

The intended function of the FBGs was to confirm that pavement slotting was relieving pavement stain as intended. In this respect, the FBGs performed their primary task very well. However, a number of additional benefits have been realised as part of this project. These benefits include:

- The ability to confirm installation location and function using real-time monitoring of live loads.

- The immediate confirmation of the impact of slotting when the pavement is under load.

- The accurate measurement of the length over which pavement slotting can relieve pavement strain and the magnitude of these movements facilitating calibration of mechanistic models.

- Improvement of the understanding of the interaction of pavement strain under thermal loading.

- Remarkable survivability considering the duration of monitoring (approaching seven years), exposure to large temperature fluctuations, lightning strikes and traffic loads as well as strains that exceeded $2 \mathrm{~mm} / \mathrm{m}$ in some locations.

Over the course of mining, the FBG data has been used extensively to better understand pavement mechanics, slot spacing and design and refine the monitoring management plan. As a measure of this success, the primary means for triggering action by the TC has been changed from ground strain to FBGs. It is envisaged that the FBGs will continue to provide insight into pavement behaviour for some time to come.

\section{Acknowledgement}

The authors would like to acknowledge BHP Billiton Illawarra Coal for funding the trial and endorsing the publication of this paper and the review by RMS Southern Region. We would also acknowledge the contribution made by staff at the Roads and Maritime Services of NSW and the entities that provide technical support and professional assistance to the TC namely MSEC, AECOM, PSM, Monitor Optics, Lynton Surveys, Colin Dove at CNS and the NSW Police Service. 


\section{References}

Buys, H, Vecovski, P, Pinkster, H, Swarbrick, G \& Kay, D 2014, 'Mining beneath the M31 Hume Motorway', in DR Kay \& G Li G (eds), Proceedings of the 9th Triennial Conference on Mine Subsidence, Mine Subsidence Technological Society, pp. 245-264.

Kay, D, Buys, H, Donald, G, Howard, M \& Pells, P 2011, 'Management of the Hume Highway pavement for subsidence impacts from longwall mining', Proceedings of the 8th Triennial Conference on Management of Subsidence Start of the Art, Mine Subsidence Technological Society, pp. 247-256.

Kay, D, Whelan, B, Donald, G \& Pinkster, H 2007, 'Monitoring mining-induced strain in a road pavement using optical fibres', Proceedings of the 7th Triennial Conference on Mine Subsidence, Mine Subsidence Technological Society, pp. 69-80.

Nosenzo, G, Whelan, BE, Brunton, M, Kay, D \& Buys, H 2013, 'Continuous monitoring of mining induced strain in a road pavement using fiber Bragg grating sensors', Photonic Sensors, vol. 3, no. 2, pp. 144-158.

Park, P, El-Tawil, S \& Park, S 2011, 'Improved geometric design of bridge asphalt plug joints', Journal of Bridge Engineering, vol. 16, no. 1, pp. 158-165.

Park, P, El-Tawil, S, Park, S \& Naaman, AE 2010, 'Behavior of bridge asphalt plug joints under thermal and traffic loads', Journal of Bridge Engineering, vol. 15, no. 3, pp. 250-259. 